\title{
Structural Determination of ATP citrate lyase
}

Nguyen, V., and Fraser, M.E.

Department of Biological Sciences, University of Calgary, Calgary, Alberta, Canada

Correspondence email: vhnguyen@ucalgary.ca

ACLY is an enzyme that is localized in the cytosol, where it catalyzes the following reaction:

$$
\text { citrate }+\mathrm{ATP}+\mathrm{CoA} \rightarrow \text { acetyl-CoA }+ \text { oxaloacetate }+\mathrm{ADP}+\mathrm{P}_{\mathrm{i}}
$$

Although the tricarboxylic acid (TCA) cycle produces acetyl-CoA, citrate synthase must convert acetyl-CoA into citrate, which can exit the mitochondria through the mitochondrial tricarboxylate transport protein because acetyl-CoA cannot be transported through the membrane. In the cytosol, ACLY converts citrate into acetyl-CoA and oxaloacetate, where the acetyl-CoA can be used for lipid biosynthesis. Since the citrate utilized comes from the catabolism of carbohydrates, ACLY links carbohydrate metabolism in the mitochondria with lipid metabolism that occurs in the cytosol. As well, ACLY may be involved in tumour development due to its role in lipid biogenesis. Thus, understanding the catalytic mechanism will reveal key features of ACLY that researchers can exploit, such as in pharmaceutical industry, or to limit or abolish the link between carbohydrates and lipids.

Structural data provides key details about an enzyme's reaction mechanism. Currently, there are structures of the amino terminal portion of ACLY, with substrates citrate, ADP- $\mathrm{Mg}^{2+}$ bound. However, no structure of the carboxyl-terminal portion is available. This is where the CoA binding site is thought to reside and where the intermediate (citryl-CoA) is cleaved to produce acetyl-CoA and oxaloacetate.

The objective is to determine the structure of the carboxyl-terminal portion of ACLY to unravel the catalytic mechanism. X-ray crystallography will be utilized for this project to obtain atomic level resolution, revealing key structural features and catalytic residues.

To isolate the carboxyl-terminal portion, the Tobacco Etch Virus (TEV) protease was used. By utilizing site-directed mutagenesis, the TEV protease recognition site (EXXYXQ S/G) was introduced by mutating several residues in the amino acid sequence of ACLY. The recognition site was mutated into various regions of ACLY, to produce four different constructs that produced a carboxyl-terminal portion of different residue length after proteolysis.

Additionally, due to the consensus amino acid sequence being EXXYXQ S/G, where proteolysis occurs between the glutamine and serine or glycine and $\mathrm{X}$ refers to any hydrophobic residue, the different constructs were made with varying residues in the $\mathrm{X}$ positions to determine their affect on protein folding and stability. The carboxyl-terminal portions can then be isolated via specific proteolysis using TEV protease, purified and then crystallized. Data from the crystals were collected from the Canadian Light Source in Saskatoon. By obtaining the structure, the reaction mechanism can be elucidated for ACLY. This could provide researchers information that can prove vital to determining ways to inhibit this enzyme. As well, this provides one step forward to solving the full-length structure of ACLY if both the amino- and carboxyl-terminal portions are available. 